NOTAS

\title{
EL PRINCIPIO DE SUBSIDIARIEDAD Y EL TRATADO DE MAASTRICHT Carlos McCadden*
}

$\mathbf{E}$ 1 Tratado de la Unión Europea es mejor conocido con el nombre de "Tratado de Maastricht" debido a que fue firmado en la ciudad holandesa de Maastricht el 7 de febrero de 1992, por los 12 países que actualmente pertenecen a la Comunidad Económica Europea. Estos 12 países (Alemania, Bélgica, Dinamarca, España, Francia, Grecia, Irlanda, Italia, Luxemburgo, Países Bajos, Portugal y el Reino Unido) acordaron la entrada en vigor de este Tratado el $1^{\circ}$ de enero de 1993, en caso de ser ratificado por los 12 , según las diversas modalidades de ratificación de cada país; sin embargo, dicha ratificación ha encontrado, como es sabido, diversos problemas.

La cuestión más importante para los Estados que ahora forman la Comunidad Económica Europea (CEE)

"Departamento Académico de Estudios Generales, ITAM. es el proyecto de la construcción de una comunidad de países unificada y el tipo de unión que desean para el futuro. Un futuro en donde la Unión Europea constituya una "Nueva Europa" o unos "Estados Unidos Europeos", creando así una unión política con un peso internacional diferente. Esta construcción empieza a tomar forma en el Tratado de Maastricht; en él las partes contratantes expresan el deseo de constituir una unión económica, monetaria y política en la cual la subsidiariedad resulta ser un principio constitucional.

En el Tratado de Maastricht el principio de subsidiariedad se trata explícitamente y es considerado como un principio constitucional para la integración europea. Esto es, precisamente por medio del principio de subsidiariedad los 12 países de la CEE buscan dar vida a una nueva forma de federalismo y a través de él se distribuirán las competencias entre la Comunidad y los Estados miembros. 


\section{NOTAS}

Este concepto permitirá que la comunidad se amplíe y se profundice, sin que se realice la pesadilla de $M$. Thatcher de "una Europa super-Estado ejerciendo un nuevo dominio desde Bruselas"(cf. Adonis/Jones, p.179).

El objeto de este artículo es estudiar el principio de subsidiariedad dentro del tratado de Masstricht y para ello lo dividiré en las siguientes partes: la primera tratará del origen y originalidad del principio de subsidiariedad; la segunda, del principio, dentro del Tratado de Maastricht; la tercera, de la distribución de competencias. Las funciones de la subsidiariedad son objeto de la cuarta parte. La subsidiariedad en relación con la soberanía y el control jurisdiccional es explicada en la quinta y sexta partes. Por último, retomo algunas ideas en la conclusión.

\section{Origen y originalidad del principio de subsidiariedad}

En su origen, la subsidiariedad no tiene nada que ver con la Comunidad Económica Europea ni con el federalismo como tal, sino que nace del catolicismo europeo de finales del siglo XIX y principios del XX, y su formulación como principio se debe al Vaticano (cf. Adonis/Jones, p.179).

Resulta sorprendente el hecho de que el origen del principio de subsidia- riedad, fundamento de la Unión Europea, no se encuentre explícitamente en ninguna de la Constituciones de los Estados firmantes de Maastricht (cf. Schindeler, p. 215). Sin embargo, esto no quiere decir que la subsidiariedad no se encuentre de una manera parcial o bajo algún aspecto en las Constituciones de varios de estos Estados cuando se hace referencia a las facultades que tiene cada Estado federado.

El principio de subsidiaridad tampoco es producto de tradición comunitaria (l'acquis communautaire), a pesar de que últimamente ha adquirido una enorme popularidad dentro del marco de la Unión Europea.

Es cierto que ya desde 1951, en el art. 5 del tratado constitutivo de la Comunidad Europea del Carbón y del Acero (CECA) se encuentran trazas de la subsidiariedad: "La Comunidad cumplirá su misión mediante intervenciones limitadas".... "Las instituciones de la Comunidad ejercerán estas actividades con un aparato administrativo reducido, en estrecha cooperación con los interesados." (TCCE, p.25 s).

El tratado de Roma (1957), que instituyó la Comunidad Económica Europea (CEE), atesta en sus artículos $114,116,215$ y 235 que la subsidiariedad se encuentra implícita en ellos aunque tienen principalmente una función procesal (cf. Constantinesco, 1, p.36).

A su vez, el Acta Única Europea (AUE) introduce en el derecho europeo originario, de manera limitada y 
NOTAS

tan sólo en materia de medio ambiente, la primera formulación de la subsidiariedad, en el art.130 R, apartado 4: "La Comunidad actuará en materia de medio ambiente en la medida en que los objetivos contemplados en el apartado 1 (= conservar, proteger y mejorar la calidad del medio ambiente; protección de la salud de las personas; garantizar una utilización prudente y racional de los recursos naturales) puedan conseguirse en mejores condiciones en el plano comunitario que en el de los Estados miembros considerados aisladamente. Sin perjuicio de determinadas medidas de carácter comunitario, los Estados miembros asumirán la financiación y la ejecución de las demás medidas" (ACTA, p.16).

1990 fue el año del (re) descubrimiento del principio de subsidiariedad; apareció en el vocabulario de la Comunidad a iniciativa del Presidente de la Comisión, Jacques Delors. (cf. Constantinesco, 1, p.35). Y no es sino hasta el Tratado de Maastricht (1992) cuando el principio de subsidiariedad se trata explícitamente y se considera como un principio constitucional de la integración europea.

Más conocido entre los teólogos que entre los políticos, este principio tiene su origen en el tomismo y su forma definitiva la adquirió en una encíclica papal.

El principio de subsidiariedad en su expresión actual tiene su origen en la Doctrina Social Católica; fue formulado por el Papa Pio XI en la encíclica
Quadragesimo Anno, en 1931, dentro del contexto de la lucha contra el totalitarismo. La encíclica de Pio XI retoma las ideas de León XIII, quien en la encíclica Rerum Novarum 40 años antes formuló los principios de la Doctrina Social de la Iglesia Católica.

Los derechos del individuo, de las sociedades intermedias y de los grupos sociales fueron defendidos en la encíclica de Pío XI contra el creciente totalitarismo de entre-guerras; esto es, frente a los regímenes fascista, socialista y comunista, que buscaban politizar o incluso aplastar a la Iglesia (cf. Adonis/Jones, p.180).

En la encíclica Quadragesimo Anno el principio de subsidiariedad es formulado así:

"Como no se puede quitar a los individuos y dar a la comunidad lo que ellos pueden realizar con su propio esfuerzo e industria, así tampoco es justo, constituyéndose un grave perjuicio y perturbación de recto orden, quitar a las comunidades menores e inferiores lo que ellas pueden hacer y proporcionar y dárselo a una sociedad mayor y más elevada, ya que toda acción de la sociedad, por su propia fuerza y naturaleza, debe prestar ayuda a los miembros del cuerpo social, pero sin destruirlos y absorberlos" (Q.A.79; p. 93).

La Doctrina Social Católica lucha contra dos concepciones extremas. Por una parte, la Iglesia se opone a la postura extrema del individualismo y rechaza el dejar todo al individuo sin 
NOTAS

intervención del Estado; la Iglesia considera al ser humano como un ser esencialmente social que necesita de los demás seres humanos. Por otra parte, la Doctrina Católica toma posición contra la postura extrema del totalitarismo, pues considera un error el que el Estado esté facultado para hacer todo, ya que los individuos, las sociedades intermedias y los diversos grupos sociales perderían así toda la eficacia de su iniciativa y de sus propios medios. Además, la Iglesia entiende que el ser humano, por ser persona, es un fin en sí mismo y no debe ser considerado como un simple medio. Un ser humano no debe ser absorbido como una pieza dentro de un todo estatal, pues perdería su ser, su iniciativa y sus propias fuerzas.

Más tarde Juan XXIII en Mater et Magistra (1961), aplica la subsidiariedad no sólo al papel de la autoridad pública dentro de la economía (estimular, regular, complementar sin remplazar), sino incluso a cuestiones de ayuda regional por parte del gobierno central.

En Pacem in Terris (1963), Juan XXIII extiende la subsidiariedad a los asuntos internacionales, los cuales por su complejidad requieren una intervención supra-nacional que no debe limitar la esfera de acción de la comunidad política nacional, y mucho menos tomar su lugar (cf.Adonis/Jones, p.182).

Pacem in Terris se hace ver que la misión propia de la "autoridad mun- dial es examinar y resolver los problemas relacionados con el bien común universal en el orden económico, social, político y cultural, ya que estos problemas, por su extrema gravedad, amplitud extraordinaria y urgencia inmediata, presentan dificultades superiores a las que pueden resolver satisfactoriamente los gobernantes de cada nación.

(141). Es decir, no corrresponde a esta autoridad mundial limitar la esfera de acción o invadir la competencia propia de la autoridad pública de cada Estado. Por el contrario, la autoridad mundial debe procurar que en todo el mundo se cree un ambiente dentro del cual no sólo los poderes públicos de cada nación, sino también los individuos y los grupos intermedios, puedan con mayor seguridad realizar sus funciones, cumplir sus deberes y defender sus derechos". (P.T. 140 y 141; p. 248)

Finalmente, se debe decir que el principio de subsidiariedad encuentra su fundamento teológico-filosófico en el tomismo. Santo Tomás defiende la tesis de que cada nivel de ser tiene su responsabilidad y sus potencialidades, las cuales deben ser respetadas y aprovechadas (cf.S.C.G.1.3,c.69-70; p. $278 \mathrm{~s})$.

\subsection{Originalidad del principio}

La subsidiariedad expresa una concepción del hombre, de la sociedad y del universo. Como principio de orga- 
NOTAS

nización social incluye primero una prohibición: todo lo que cada uno puede realizar por sí mismo y con sus propias fuerzas no debe ser transferido a otro nivel. Pero tiene sobre todo un sentido positivo: cuando la capacidad de una acción comunitaria se revela insuficiente no debe ser tomada a cargo automáticamente dentro de una comunidad más amplia. Al contrario, esta comunidad más amplia deberá ayudar y sostener a la comunidad deficiente. Desde su nivel superior debe restaurar la capacidad de acción de la comunidad que resulta momentáneamente insuficiente. Es aquí donde se revela la etimología del término subsidiariedad: subsidio (subsidium), auxilio (cf. Constantinesco, 1, p. 212), que significa ayuda o soporte y no substitución (Adonis/Jones, p.183).

\subsection{Subsidiariedad y federalismo}

La cuestión que surge es la de si un principio teológico-filosófico puede tener un contenido jurídico como para llegar a ser uno de los principios constitucionales de la integración europea. A la vez, habría que saber si de alguna manera este principio añade algo a la concepción federalista tradicional.

La originalidad de la subsidiariedad se ve claramente comparándola con el federalismo. Según Schindler (p.215), el federalismo y la subsidiariedad no se encuentran necesariamente relacionados; el federalismo no está forzosamente condicionado por la preeminencia de las sociedades más pequeñas sobre las más amplias.

La concepción federalista tradicional, nos dicen Adonis y Jones (p.193), incluye por lo menos tres ideas: una división específica de competencias, disposiciones para el ejercicio de la autoridad en áreas de competencias concurrentes, y una cláusula reservando poderes sin trabas a los Estados para actuar en todas las áreas no reservadas, única o concurrentemente, a la federación.

Por su parte, la subsidiariedad no es una fórmula neutral de la distribución del poder dentro de la sociedad; al contrario, gira en torno a una determinada concepción del orden social, subrayando al máximo la auto-ayuda (cf. Adonis/Jones, p. 183). En cambio, la concepción federalista no involucra necesariamente la idea de que lo que el individuo y las sociedades o asociaciones intermedias pueden realizar por su propia iniciativa y con sus propias fuerzas deba ser realizado por ellos mismos, a riesgo de resultar injusto un orden en el que una sociedad más amplia asuma esas facultades. Así, para efectuar la división de competencias entre la Comunidad y los Estados miembros, la subsidiariedad resulta ser un critero determinado que, sin estar contenida en ella, se puede añadir a la noción de federación.

De los 12 países de la Comunidad es sobre todo en la doctrina constitucional alemana en donde el principio 
NOTAS

de subsidiariedad ha sido formulado $y$ desarrollado más ampliamente; en ella la subsidiaridad tiene principalmente tres funciones (cf. Constantinesco, 2, p. $37 \mathrm{~s} .:)$ a) Es entendida como un principio de ética política; considera que la sociedad no está principalmente formada por individuos sino por comunidades diversas, dentro de las cuales se sitúa aquél para permitirle su pleno desarrollo. En esta concepción organicista de la sociedad se le reconoce una preeminencia a las comunidades más simples, las cuales deben realizar todas las tareas que les permitan sus propias fuerzas. b) Es también un principio de repartición de competencias, en el sentido de que es por medio de la subsidiariedad como se distribuyen las competencias entre los diferentes niveles de organización pública; cada nivel de poder no debe tener competencia para realizar sino lo que mejor pueda realizar.c) Es, por último, un principio que interviene en el ámbito de las competencias concurrentes. Permite la actualización de una competencia potencial de la Federación; si ésta puede intervenir mejor que los Estados (Laender), entonces debe hacerlo, aunque los Estados ya hayan tomado, cada uno independientemente, las medidas apropiadas. De este modo, en el derecho constitucional alemán la subsidiariedad resulta ser un principio multifuncional, político y jurídico, que proteje a las personas en el seno de diversas comunidades.

\subsection{Lo que no es la subsidiariedad}

A. Faber (cf. p.1134) hace notar que el principio de subsidiariedad no debe confundirse con el principio de efectividad o de optimización (Effektivitätsprinzip oder Optimierungsgebot). Aquél determina la intervención de la unidad superior sólo en el caso de que la tarea respectiva de la unidad inferior no pueda ser observada. En cambio, el principio de efectividad u optimización le reconoce competencia a la unidad superior cuando la tarea puede ser "mejor" llevada a cabo. La diferencia consiste en que en el caso de la subsidiariedad, la competencia de la esfera inferior tiene un valor en sí misma; en cambio en el principio de efectividad la esfera inferior padece un menoscabo en la ejecución de su competencia puesto que es substituida.

Por otra parte, el principio de subsidiariedad no debe entenderse como un principio útil para resolver únicamente un problema finaciero. $\mathrm{Ha}$ habido sugerencias (Contanstinesco habla del caso de España; cf. 2, p. 226) en el sentido de que las tareas que la Comunidad se proponga realizar a nombre de la subsidiariedad sean realizadas solamente si ésta tiene medios financieros suficientes que permitan llevarlas a cabo. Esta manera de entender la subsidiariedad desconoce el hecho de que la actividad de la Comunidad es esencialmente normativa más que operacional o financiera. 
NOTAS

2. El principio de subsidiariedad en el Tratado de la Unión Europea firmado en Maastricht

El Tratado de Maastricht asigna objetivos a la Comunidad y señala, a la vez, que la Comunidad actuará dentro de los límites de las competencias que el Tratado le atribuye. En él las relaciones entre las competencias nacionales y comunitarias deberán ser regidas y equilibradas por el principio de subsidiariedad. En la delimitación de los ámbitos de competencia, el Tratado hace referencia expresa, en el artículo $3 \mathrm{~B}$, párrafo segundo, al principio de subsidiariedad de la manera siguiente:

"En los ámbitos que no sean de su competencia exclusiva, la Comunidad intervendrá, conforme al principio de subsidiariedad, sólo en la medida en que los objetivos de la acción pretendida no puedan ser alcanzados de manera suficiente por los Estados miembros y, por consiguiente, puedan lograrse mejor, debido a la dimensión o a los efectos de la acción contemplada, a nivel comunitario" (TRATADO, p.13 s.).

Dos observaciones:

(a) Resulta interesante la elección que se hace en el texto "versión castellana" (que coincide con la alemana pero no con la francesa en este punto) al formular de una manera positiva este principio. Constantinesco ya había hecho notar (cf.1, p.220) que en la formulación existen dos alternativas: la positiva ("la Comunidad intervendrá en la medida..."), la cual deja relativamente abiertas las posibilidades de la Comunidad; o la negativa ("la Comunidad no intervendrá sino y tan sólo en la medida en que..."), que restringe las posibilidades de la Comunidad y que obligaría a las instituciones comunitarias que quieran hacer valer la subsidiariedad a aportar una prueba más exigente. Ambas formulaciones muestran las dos facetas de la subsidiariedad, pero la positiva, que es la que parece tomar el texto, fomenta el desarrollo de la Comunidad.

(b) El a.3.B incluye una formulación que se asemeja más al principio de efectividad u optimización que al de subsidiariedad (cf. Faber, p.1135), o al menos se trata de un híbrido que incluye en su formulación a ambos.

El a.3.B aunque habla explícitamente del principio de subsidiariedad, no considera a los Estados miembros como sociedades inferiores o subordinadas y no hace mención directamente a la relación entre las sociedades inferiores y las superiores. En cambio, sí incluye el término "mejor", que es propio del principio de efectividad u optimización. 
NOTAS

Si se quiere conservar el sentido original del principio de subsidiariedad, la interpretación y eventualmente la jurisprudencia deberán acentuar la prohibición que hace el principio, en el sentido de que todo lo que cada uno puede realizar por sí mismo y con sus propias fuerzas no debe ser transferido a otro nivel. El problema se encuentra en la interpretación que se pueda hacer del término "mejor" que contempla el artículo. El riesgo es que se puede cambiar de principio y en lugar de fortalecer las estructuras locales y estatales lo que se fortalecería sería un tipo de centralismo europeo.

Un principio así formulado difícilmente puede estar sujeto a un control jurisdiccional, pues la cuestión de saber si algo se puede llevar a cabo "mejor" por medio de la Comunidad que por medio de la acción particular de los Estados miembros es de naturaleza política y no jurídica.

El texto ("la Comunidad intervendrá"..."sólo en la medida en que los objetivos de la acción pretendida no puedan ser alcanzados de manera suficiente por los Estados miembros y, por consiguiente, puedan lograrse mejor"... "a nivel comunitario") deberá entenderse en el sentido de que si la capacidad de acción de los Estados miembros (esfera inferior) se revela insuficiente, ésta no debe ser tomada a cargo automáticamente por la Comunidad. Sino al contrario, la Comunidad, por ser más amplia, deberá ayudar y sostener a los Estados a al- canzar los objetivos de la acción pretendida, respetando su naturaleza.

\section{La subsidiariedad como principio de atribución de competencias}

La subsidiariedad permite determinar las competencias de los diferentes niveles de la organización pública de los Estados miembros y de la Comunidad. Este principio puede ser utilizado fecundamente tanto en la atribución de competencias entre las esferas infraestatales y las estatales como entre las esferas estatales y las supra-estatales (cf. A.Faber, p.1134). Heintzen (cf. p. $318,320 \mathrm{~s}$.) hace ver que la lógica del principio permite la asignación de competencias a las instancias inferiores dentro de un Estado (como en el caso de los Laender en Alemania, cf. A. Faber, p. 1134) y a la vez puede servir para atribuir competencias a la Unión, que es superior al Estado.

Piedra angular dentro de la ordenación de competencias europeas, la subsidiariedad presenta la desventaja de que la determinación de las competencias parece imponerse casi por sí misma. Sin llegar al extremo de esta ilusión irracional, queda sin embargo la certeza de que existe un criterio definido dentro de la difícil tarea de la atribución y transferencia de competencias entre los Estados y la Comunidad (cf. Constantinesco, 1, p.213). Este criterio se opone a cualquier ten- 
dencia centralista que la Comunidad pudiera empezar a desarrollar.

El Tratado de Maastricht muestra el producto de la difícil tarea de trasladar las competencias nacionales a la esfera comunitaria. Pero no propone una Comunidad tendiente a absorber las competencias estatales y que luego en un gesto amable devuelva algunas. En el Tratado la subsidiariedad no se confunde con el principio de atribución de competencias, pues aunque la técnica de devolución de competencias muestra su carácter subsidiario, no por ello se confunden estas dos categorías (cf. Constantinesco, 1, p. 215).

\subsection{Las competencias concurrentes}

Ya desde el Acta Única Europea (AUE), con la formulación de la subsidiariedad en el ámbito del medio ambiente es contemplada una situación particular sobre el plano de las competencias, que hace referencia al caso de las llamadas competencias concurrentes. En el Acta Única se enuncian los criterios según los cuales la Comunidad podría ejercer una acción (cf. Constantinesco, 1, p. 218).

Pero no es sino hasta el Tratado de Maastricht cuando el principio de subsidiariedad adquiere un carácter normativo (cf.Heintzen, p. 318) al ser integrado en el texto mismo del Tratado; en él el principio de subsidiariedad resuelve el caso de competencias con- currentes, adquiriendo una dimensión general en el ámbito de todo el Tratado.

En el Tratado la subsidiariedad resulta ser operativa. La Comunidad puede por este medio actualizar una competencia potencial cada vez que, dentro de una materia determinada, la competencia se encuentre compartida entre los Estados miembros y la Comunidad. Pero para ello deberá probar, de acuerdo con el art.3 B, par. 2, que "los objetivos de la acción pretendida no puedan ser alcanzados de manera suficiente por los Estados miembros, y por consiguiente, puedan lograrse mejor, debido a la dimensión o a los efectos de la acción contemplada, a nivel comunitario" (Tratado, p. $13 \mathrm{~s}$.). Esto es válido incluso en el caso de que los Estados miembros ya hayan tomado, cada uno independientemente, las medidas que consideren apropiadas.

El principio será ciertamente utilizado como un arma dentro de la lucha política, cuando cada uno de los Estados miembros busquen defender sus respectivas competencias; sin embargo, la subsidiariedad garantiza al menos que la consistencia de las competencias de la Comunidad será respetada cuando ésta actúa en los ámbitos que le corresponden. 
NOTAS

\subsection{Funciones de la subsidiariedad}

Según la opinión de Constantinesco (cf. 2, p. 39 s.), la subsidiariedad tiene dos funciones: una como principio de legitimación política y otra como principio operacional.

a) Como principio de legitimación política la subsidiariedad llega a obtener un enorme consenso, pues propone una Comunidad que concentra sus esfuerzos en ciertas áreas delimitadas en donde su acción es más eficaz que la de los países miembros. En este sentido la subsidiariedad ha sido una respuesta política a una inquietud política, puesto que no se trata de una Comunidad que busca concentrar el poder vaciando las competencias de los Estados miembros.

La subsidiariedad, por otra parte, refuerza una tendencia observada desde hace más de diez años, en el sentido de una mayor atención a las colectividades públicas infraestatales. La subsidiariedad introduce una nueva idea de distribución de poder entre la Comunidad, el Estado y la región, eliminando el problema de que la Comunidad pueda aspirar a usurpar competencias que las Constituciones nacionales han reconocido o devuelto a las entidades infraestatales. Aquí la subsidiariedad se convierte en un principio que adquiere una doble función: puede ser utilizado para la integración comunitaria y para la regionalización de los Estados miembros (cf. Heintzen, p. 321). Esto debe desembocar en una nueva relación entre las entidades infra-estatales y la Comunidad. De hecho, antes de Maastricht (cf.Constantinesco, 1, p. 222 s.), ya se había hablado de una representación específica de las entidades regionales, ya sea como un partido en el Parlamento Europeo o como un Consejo de Regiones, cuyos poderes aún estarían por determinarse.

El problema que por otra parte plantea esta nueva relación es que se relativiza el monopolio de la representación internacional de un Estado por medio de su Gobierno, y esto al grado de que se puede uno preguntar si la integración comunitaria no diluirá las fronteras, aparentemente rígidas, que separan el orden interno del orden internacional.

b) Como principio operacional, la subsidiariedad limita las competencias de la Comunidad a las áreas en que ésta pueda probar que su intervención está justificada. Se trata, en cierto sentido, de un desplazamiento de la carga de la prueba que muestra que la acción de la Comunidad no debe substituir a la de los Estados, sino completarla y enriquecerla de una 
NOTAS

manera cualitativa (cf. Constantinesco, 1, p. 218). La subsidiariedad resulta así un mecanismo para determinar la carga de la prueba, puesto que corresponde a la Comunidad demostrar que su nivel de decisión es más eficaz para resolver un problema cuya naturaleza exige una decisión común. Así la Comunidad podrá ejercitar una competencia potencial, sólo si puede aportar la prueba de que su acción está justificada.

\section{Soberanía y Subsidiariedad}

La subsidiariedad parece modificar la noción clásica de Estado moderno, donde éste es soberano. El modelo de los Estados miembros de la Comunidad exige una evolución en la cual se llegue a una nueva noción de soberanía. Esta nueva noción comprenderá una co-participación y una co-decisión supraestatal, que exige una racionalización de la gestión pública.

Con la ayuda de la subsidiariedad se busca una nueva localización de las competencias y una distribución adecuada de los poderes entre los diversos escalones de la organización politico-administrativa, que permita responder al problema de quién es competente y para qué a nivel de la Comunidad, los Estados miembros y las regiones (cf. Constantinesco, 1, p. 210), con lo que la subsidiariedad se convierte en una garantía para los go- biernos temerosos de los abusos que pueda hacer Bruselas de su "soberanía" (Adonis/Jones, p.184). Además permite sobre todo ordenar legalmente una situación de hecho, puesto que actualmente ninguno de los Estados europeos es absolutamente soberano. Un Estado miembro de una Comunidad, apoyado en el argumento de la subsidiariedad, puede defender mejor sus competencias ante los ataques de la Comunidad que en una Comunidad sin dicho principio y en donde el Estado tan sólo puede apelar a la soberanía estatal. El argumento de la soberanía puede más facilmente ser atacado de subjetivo que el argumento de la subsidiariedad, el cual ofrece un criterio objetivo para la distribución de competencias (cf. Heintzen, p. 319).

\section{Subsidiariedad y control jurisdic- cional}

El principio de subsidiariedad de Maastricht puede ser sometido a una instancia controladora que verifique que los criterios establecidos han sido correctamente utilizados. Ante una cuestión tan delicada habría que preguntarse si el control que se ejercerá será puramente jurídico o también político.

En el caso de un control jurisdiccional, la Corte de Justicia de la Comunidad se reconocería competente para 
NOTAS

conocer sobre este principio. Así el principio podría ser invocado delante de un tribunal para apoyar una demanda y la Corte tendría que juzgar si la interveción comunitaria es mejor que lo que podría lograr un país actuando separadamente (cf. Constantinesco, 2, p. $42 \mathrm{~s}$ ).

Por el contrario, un control puramente político sería, por ejemplo, el que pudiera ejercer un cuerpo o un órgano no jurisdiccional. Un cuerpo de hombres sabios (wise men) ha sido sugerido (cf. Adonis/Jones, p.186); sin embargo, esto complicaría la estructura institucional de la Comunidad, y además añadiría confusión a la distribución de autoridad y de responsabilidades, pues aparecería otra institución con poder de decisión.

En el contexto del Tratado de Maastricht el principio de subsidiariedad queda sometido al control jurisdiccional de la Corte de Justicia; y en el caso de que el control fuera puramente jurídico, la Corte de Justicia tendría además, en cierto sentido, la misión de una corte constitucional (cf. Constantinesco, 2, p. 43).

El control jurisdiccional podría resultar muy eficaz contra las competencias exageradas que pudiera generar el voto por mayoría calificada. Pero, por otra parte, se corre el riesgo de consignar los problemas políticos controvertidos a la Corte para que ésta los determine (Adonis Jones, p.186).

Por último, habría que decir que si por virtud de alguna cláusula la subsi- diariedad no estuviera sometida a un control jurisdiccional, entonces se comprometería la integridad de la Unión y resurgiría el peligro del centralismo.

\section{Conclusión}

La subsidiariedad resulta ser un principio con dos caras. Por el lado de la Comunidad, permitirá que ésta crezca de una manera justificada y racional; $y$ por el lado de los Estados miembros, éstos podrán reivindicar una mejor asociación a la gestión de las competencias transferidas a la Comunidad. Esta doble naturaleza de la subsidiariedad permite entender también el porqué de la popularidad de este principio, pues tanto los países miembros como la Comunidad pueden invocarlo razonablemente a su favor (cf. Constantinesco, 1, p. 209).

Sin embargo, tal como quedó formulado en el artículo $3 \mathrm{~B}$, párrafo segundo, el principio de subsidiariedad del Tratado de Maastricht se asemeja más al principio de efectividad u optimización que al principio de subsidiariedad de la Doctrina Social Católica, a pesar de que en el texto se hace mención expresa de la subsidiariedad.

Debido a la inexactitud de la formulación, queda sujeto a la interpretación, y eventualmente a la jurisprudencia, el acentuar que cuando la capacidad de acción de los Estados miembros se revela insuficiente, 
NOTAS

ésta no deberá ser tomada a cargo automáticamente por la Comunidad, sino que la Comunidad deberá sostener y ayudar a los Estados a alcanzar los objetivos de la acción pretendida.

El riesgo es que se puede cambiar fácilmente de principio y la nueva Unión, en vez de fortalecer las estructuras locales, lo que fortalecería sería una modalidad de centralismo europeo.

\section{Bibliografía}

- Andrew Adonis/Stuart Jones, Subsidarity and the European Community's Constitutional Future, Staatswissenschaften und Staatspraxis 1991/1992, Heft, 2, p.179-196.

- Constantinesco, Vlad, 1, La subsidiarité comme principe constitutionnel de l'integration européenne; Aussenwirtschaft: Schweizerische Zeitschrift für internationale Wirtschaftbeziehungen. Oktober 1991, p.439-459. (S. 207 des Sondersheftes).

- Constantinesco, Vlad, 2, Le principe de Subsidiarité: un passage obligé vers l'union européenne? L'Europe et le droit. Mélanges en hommage à Jean Boulouis. Editions Dalloz. p. 37-45.

- Angela Faber, Die Zukunft kommunaler Selbstverwaltung und der Gedanke der Subsidiarität in den Europäischen Gemeinschaften, Deutsches Verwaltungsblatt 1991, p.1126 s.

- Markus Heintzen, Subsidiaritätsprinzip und Europäische Gemeins- chaft, (Deutsche) Juristen Zeitung 1991, p. $317 \mathrm{~s}$.

- Dietrich Schindler, Schweizerischer und europäischer Föderalismus. Schweizerisches Zentralblatt für Staatsund Verwaltungsrecht. Band 93 N. 5, Mai 1992. p.193-223.

\section{Abreviaturas:}

ACTA. Acta Única. Boletín de las Comunidades Europeas. Suplemento 2/86. 1986. Luxemburgo: Oficina de Publicaciones Oficiales de las Comunidades Europeas.

P.T. Pacem in Terris. (Juan XXIII) En: Ocho grandes mensajes, B.A.C., Madrid, 1974, p. 201-258.

Q.A. Quadragesimo Anno. (Pio XI). En: Ocho grandes mensajes, B.A.C., Madrid, 1974, p. 57-120.

S.C.G. Suma Contra Gentiles, Tomas de Aquino, B.A.C., Madrid, 1968.

TCCE. Tratados constitutivos de las Comunidades Europeas. (CECA, CEE, CEEA). Acta Única Europea. Textos relativos a las Comunidades. Edición abreviada, 1987, Luxemburgo: Oficina de Publicaciones Oficiales de las Comunidades Europeas.

TRATADO. Tratado de la Unión Europea, 1992, Luxemburgo: Oficina de Publicaciones Oficiales de las Comunidades Europeas. 